\title{
Unexplained stillbirth versus SIDS: Common congenital diseases of the autonomic nervous system-pathology and nosology
}

\author{
Luigi Matturri*, Anna M. Lavezzi \\ "Lino Rossi" Research Center for the Study and Prevention of Sudden Perinatal Death and SIDS, Department of Surgical, Reconstructive and Diagnostic Sciences, University of Milan, \\ Milan 20122, Italy
}

\section{A R T I C L E I N F O}

Article history:

Received 3 November 2010

Received in revised form 10 December 2010

Accepted 20 December 2010

\section{Keywords:}

Stillbirth

SIDS

Autonomic nervous system

Cardiac conduction system

Neuropathology

\begin{abstract}
A B S T R A C T
Objective: To contribute to a more balanced assessment of the morphological substrates underlying unexplained perinatal death and SIDS.

Methods: In-depth histological, immunohistochemical and genetic examinations were performed on the autonomic nervous and cardiac conduction systems in 95 unexpected perinatal deaths, 140 SIDS and 78 controls (44 infants and 34 perinatal death victims).

Results: The study revealed the localization and the nature of a variety of specific congenital abnormalities of the autonomic nervous system, central and peripheral, and of the cardiac conduction system that represent the morphological substrates of the pathophysiological mechanism of sudden fetal death and SIDS.

Conclusions: The observation of similar anomalies of the autonomic nervous and the cardiac conduction systems in both unexplained perinatal deaths and SIDS indicates their common congenital nature. Therefore, the definitions of these deaths, currently nosographically distinct, should be unified.
\end{abstract}

(c) 2010 Elsevier Ireland Ltd. All rights reserved.

\section{Introduction}

Perinatal loss and the Sudden Infant Death Syndrome (SIDS) are still an unresolved, major social and health problem today [1].

This paper will tackle unexplained stillbirth and early neonatal deaths, as well as the SIDS, which are apparently accounted for by exclusively or preeminently neurovegetative abnormalities which were not suspected during prenatal clinical examinations. So far, only a few studies have made a close examination of the nervous system, although abnormalities of this system are obviously extremely relevant to any analysis aiming to gain a better understanding of unexplained death during gestation and in early infancy. Thus, today's basic information in this field is still inadequate [2-5].

To contribute to a more balanced assessment of the morphological substrates underlying unexplained perinatal death and SIDS, the present article will focus upon the multifaceted involvement of the central and peripheral autonomic nervous system, as well as the cardiac conduction system, subject to autonomic nervous system control.

The results presented herein, obtained from in-depth histological examinations of the autonomic nervous and cardiac conduction systems in a very wide sample of unexpected perinatal deaths (65 stillbirths and 30 early neonatal deaths), SIDS (140 victims) and 78

\footnotetext{
* Corresponding author. Lino Rossi" Research Center for the Study and Prevention of Sudden Perinatal Death and SIDS, University of Milan, Italy, Via della Commenda, 19, 20122 Milan, Italy. Tel.: +39 02 50320800; fax: +39 0250320823.

E-mail address: luigi.matturri@unimi.it (L. Matturri).
}

controls (44 infants and 34 perinatal death victims), show similar alterations in unexplained death victims, indicating their common congenital nature and then that unexplained fetal and early neonatal death should not be regarded as distinct from the SIDS.

\section{Materials and methods}

\subsection{Study subjects}

The study included 313 subjects. This was a selected set of cases, sent to our Research Center according to the application of the guidelines recognized by Italian law n.31 "Regulations for Diagnostic Post Mortem Investigation in Victims of SIDS and Unexpected Fetal Death" over a 9-year period (2000-2009). This law decrees that all infants suspected of SIDS who died suddenly in Italian regions within the first year of age, as well as all fresh fetuses who died after the 25th week of gestation without any apparent cause, must undergo an in-depth anatomo-pathological examination. The autopsy was performed in all cases according to the International Standardised Autopsy Protocol (ISAP) of the Global Strategy Task Force of SIDS International [6], and the neuropathologic protocol developed at the Authors' research center $[7,8]$. These guidelines include all the methodologies for the study of the central and peripheral autonomic nervous system and of the cardiovascular system.

Below we briefly summarize the protocol for the examination of the brainstem, the spinal cord and the cerebellum, where the main structures participating in control of the vital functions (cardiorespiratory, arousal, 
upper digestive tract, etc.) are located. The other methodologies are available in the above-mentioned references $[7,8]$.

Fresh specimens were firstly collected from the brainstem, near the obex, and conserved in ethanol or in RNA-later reagent (AMBION, Inc; Austin, TX) for genetic studies of the serotonin transporter polymorphism that has been widely associated to SIDS [9], and of the PHOX2B gene, whose mutation causes a large decrease in the central chemoreflex responsible for the Congenital Central Hypoventilation Syndrome (CCHS) [10].

After fixation in $10 \%$ phosphate-buffered formalin, the brainstem, the spinal cord and cerebellum were processed and paraffin-embedded. Transverse serial sections of the midbrain, pons, medulla oblongata, thoracic spinal cord and cerebellar hemispheres were made at intervals of 50-60 $\mu \mathrm{m}$. For each level, serial $5 \mu \mathrm{m}$ sections were obtained, two of which were routinely stained for histological examination using hematoxylin-eosin and Klüver-Barrera and the remaining sections were submitted to immunohistochemical study of neurotransmitters such as somatostatin, serotonin, tyrosine-hydroxylase, or specific visualization of apoptotic cells and reactive astrocytes through glial fibrillar acidic protein (GFAP) method.

The routine histological evaluation of the brainstem was focused on the locus coeruleus and the parabrachial/Kölliker-Fuse complex in the rostral pons/caudal mesencephalon, on the retrotrapezoid nucleus, the superior olivary complex and the facial/parafacial complex in the caudal pons; on the hypoglossus, the dorsal motor vagal, the tractus solitarius, the ambiguus, the pre-Bötzinger, the inferior olivary, the raphé and the arcuate nuclei in the medulla oblongata. In the thoracic spinal cord the intermediolateral nucleus was the subject of analysis. In the cerebellum, the cortex layers (external granular layer, molecular layer, Purkinje cell layer and internal granular layer) and the medullary deep nuclei (the dentate nucleus, the fastigial nucleus, the globose nucleus and the emboliform nucleus) were examined.

In 235 cases, after the in-depth anatomopathological examination, the death remained totally unexplained. A diagnosis of "unexplained perinatal death" was established for 65 stillbirth cases (30 females and 35 males, aged 24-40 gestational weeks; median age: 38 weeks; 49 ante-partum deaths and 16 intra-partum deaths) and 30 early neonatal death victims ( 16 females and 14 males, who died in the first days of life) and of "SIDS" for 140 infants, 66 females and 74 males, aged from 1 to 10 postnatal months (median age: 3.3 months) or more precisely, from 35 to 73 postconceptional weeks (median age: 46 postconceptional weeks).

In the remaining 78 cases, 44 infants (16 females, 23 males; aged from 2 to 8 postnatal months, median age: 3 months) and 34 perinatal death victims ( 15 females, 19 males aged from 25 gestational weeks to 7 days of postnatal life; median age: 36 gestational weeks), a precise cause of death was formulated at autopsy (adnexa pathologies and cardiomyopathies in perinatal deaths; cardiomyopathies and pneumonia in infant deaths). These cases were used as "Controls."

The histological analyses were carried out by two independent and blinded observers. Comparison among diagnoses was performed employing Kappa statistics (Kappa Index-KI) to evaluate the interobserver reproducibility. The inter-observer reproducibility assessed throughout the study was very satisfactory $(\mathrm{KI}=0.85)$. Moreover, in case of disagreement between the investigators, the slides were reviewed and discussed until the same results were obtained.

\subsection{Statistical analysis}

The statistical significance of direct comparisons between groups of victims (unexplained perinatal and infant deaths) was determined using the Levene test, one way analysis of variance (ANOVA) and Student's $t$-test. The selected threshold level for statistical significance was $p<0.05$.
For each case, all available information about pregnancy, fetal development and delivery and, in cases of infant death, about the environmental and familial situation where the death occurred, besides information related to the potential risk factors (such as maternal smoking, maternal obesity, type of milk feeding, position the baby was last left in), were collected and categorised during post-mortem family interviews.

All the information sheets were recorded in the registry of a dedicated data bank, administered by the Health Government of the Lombardy Region, and established under two subsections: one for perinatal loss (unexplained stillbirth, early neonatal death and perinatal controls) and another for the SIDS [11].

\section{Results}

We firstly analyzed the distribution of different information extracted from the data bank related to 129 perinatal deaths (65 sudden fetal deaths, 30 sudden early neonatal deaths and 34 control perinatal deaths) and 184 infant deaths (140 SIDS and 44 controls) to evaluate the potential risk factors. Tables 1 and 2 display the rates and percentage distributions of these variables related to perinatal and infant deaths, respectively. Significant correlations $(p<0.05)$ were observed between maternal smoking, maternal obesity, brain weight below the normal value and sudden unexplained perinatal deaths (Table 1), and between prematurity, maternal smoking and the SIDS (Table 2). Data related to maternal abuse of alcohol, drugs, or sedative drugs were not available.

All the pathologic results revealed by in-depth anatomopathological examinations are summarized in Table 3. A more in-depth description of the neuropathologic findings and the related illustrations can be found in our previous works [12-18].

Table 1

Distribution of potential risk factors for unexplained perinatal deaths (65 stillbirths and 30 early neonatal deaths) and controls (34 cases) - (total number of victims: 129).

\begin{tabular}{|c|c|c|}
\hline \multirow[b]{2}{*}{ Total number } & \multirow{2}{*}{$\begin{array}{l}\begin{array}{l}\text { Unexplained perinatal } \\
\text { deaths }\end{array} \\
95 \text { (rate) }\end{array}$} & \multirow{2}{*}{$\begin{array}{l}\text { Explained perinatal } \\
\text { deaths } \\
34 \text { (rate) }\end{array}$} \\
\hline & & \\
\hline \multicolumn{3}{|l|}{ Age } \\
\hline$\leq 35$ gestational weeks & $35(36.8 \%)$ & $16(47.0 \%)$ \\
\hline$>35$ gestational weeks & $60(63.2 \%)$ & $18(53.0 \%)$ \\
\hline \multicolumn{3}{|l|}{ Sex } \\
\hline Male & $49(51.6 \%)$ & $19(55.9 \%)$ \\
\hline Female & $46(48.4 \%)$ & $15(44.1 \%)$ \\
\hline \multicolumn{3}{|l|}{ Race } \\
\hline White & $73(76.8 \%)$ & $28(82.3 \%)$ \\
\hline Others & $22(23.2 \%)$ & $6(17.7 \%)$ \\
\hline \multicolumn{3}{|l|}{ Weight } \\
\hline Normal value for age & $36(37.9 \%)$ & $18(52.9 \%)$ \\
\hline Below normal value for age & $59(62.1 \%)$ & $16(47.1 \%)$ \\
\hline \multicolumn{3}{|l|}{ Brain weight $\left({ }^{*}\right)$} \\
\hline Normal value for age & $32(33.7 \%)$ & $24(70.6 \%)$ \\
\hline Below normal value for age & $63(66.3 \%)$ & $10(29.4 \%)$ \\
\hline \multicolumn{3}{|l|}{ Adnexa (placenta-umbilical cord) } \\
\hline Normal structure & 75 (71.2\%) & $22(64.7 \%)$ \\
\hline Pathological structure & $20(28.8 \%)$ & $12(35.3 \%)$ \\
\hline \multicolumn{3}{|l|}{ Maternal age } \\
\hline$\leq 30$ years & $33(34.7 \%)$ & $14(41.1 \%)$ \\
\hline$>30$ years & $62(65.3 \%)$ & $20(58.9 \%)$ \\
\hline \multicolumn{3}{|l|}{ Maternal weight $\left({ }^{*}\right)$} \\
\hline Normal value for age & $50(52.6 \%)$ & $28(82.3 \%)$ \\
\hline Above normal value for age (Obesity) & $45(47.4 \%)$ & $6(17.7 \%)$ \\
\hline \multicolumn{3}{|c|}{ Maternal smoking $\left(^{*}\right)$} \\
\hline No & $50(52.6 \%)$ & $29(85.3 \%)$ \\
\hline Yes & $45(47.4 \%)$ & $5(14.7 \%)$ \\
\hline
\end{tabular}

$\left({ }^{*}\right)$ When comparing unexplained perinatal deaths with the control group, $p<0.05$. 
Table 2

Distribution of potential risk factors for SIDS (140 cases) and controls ( 44 cases) - (total number of victims: 184).

\begin{tabular}{|c|c|c|}
\hline \multirow[b]{2}{*}{ Total number } & \multirow{2}{*}{$\begin{array}{l}\text { Unexplained infant deaths } \\
\text { (SIDS) } \\
140 \text { (rate) }\end{array}$} & \multirow{2}{*}{$\begin{array}{l}\begin{array}{l}\text { Explained infan } \\
\text { deaths }\end{array} \\
44 \text { (rate) }\end{array}$} \\
\hline & & \\
\hline \multicolumn{3}{|l|}{ Postnatal age } \\
\hline$\leq 4$ months & $115(82.1 \%)$ & $37(84.1 \%)$ \\
\hline$>4$ months & $25(17.9 \%)$ & $7(15.9 \%)$ \\
\hline \multicolumn{3}{|l|}{ Postconceptional age } \\
\hline$\leq 52$ weeks & $123(87.8 \%)$ & $39(88.6 \%)$ \\
\hline$>52$ weeks & $17(12.2 \%)$ & $5(11.4 \%)$ \\
\hline \multicolumn{3}{|l|}{ Gestational age at birth $\left(^{*}\right)$} \\
\hline$\leq 37$ weeks (prematurity) & $76(54.2 \%)$ & $6(13.6 \%)$ \\
\hline$>37$ weeks & $64(45.8 \%)$ & $38(86.4 \%)$ \\
\hline \multicolumn{3}{|l|}{ Sex } \\
\hline Male & $74(52.8 \%)$ & $26(59.0 \%)$ \\
\hline Female & $66(47.2 \%)$ & $18(41.0 \%)$ \\
\hline \multicolumn{3}{|l|}{ Birth weight } \\
\hline normal value for gestational age & $56(40 \%)$ & $14(31.8 \%)$ \\
\hline below normal value for gestational age & $84(60 \%)$ & $30(68.2 \%)$ \\
\hline \multicolumn{3}{|l|}{ Brain weight } \\
\hline normal value for age & $94(67.1 \%)$ & $26(59.0 \%)$ \\
\hline below normal value for age & $46(32.9 \%)$ & $18(41.0 \%)$ \\
\hline \multicolumn{3}{|l|}{ Race } \\
\hline White & $112(80.0 \%)$ & $33(75.0 \%)$ \\
\hline Others & $28(20.0 \%)$ & $11(25.0 \%)$ \\
\hline \multicolumn{3}{|l|}{ Maternal smoking $\left({ }^{*}\right)$} \\
\hline no & $81(57.8 \%)$ & $34(77.3 \%)$ \\
\hline yes & $59(42.2 \%)$ & $9(22.7 \%)$ \\
\hline \multicolumn{3}{|l|}{ Feeding } \\
\hline human milk & $71(50.7 \%)$ & $19(43.2 \%)$ \\
\hline formula/mixed & $69(49.3 \%)$ & $25(56.8 \%)$ \\
\hline \multicolumn{3}{|l|}{ Position last left } \\
\hline prone & $75(53.6 \%)$ & $25(56.8 \%)$ \\
\hline supine & $65(46.4 \%)$ & $19(43.2 \%)$ \\
\hline
\end{tabular}

$\left({ }^{*}\right)$ When comparing unexplained infant deaths with the control group, $p<0.05$.

\subsection{Neuropathologic findings in stillbirth versus SIDS}

\subsubsection{Autonomic central nervous system}

- Morphological alterations

In the brainstem the main structural alterations were hypodevelopment of the different nuclei and structures.

In the medulla oblongata hypoplasia of the arcuate nucleus was present in over $50 \%$ of the sudden perinatal deaths and SIDS victims. In detail, in $36 \%$ of the cases the hypoplasia was bilateral, extending throughout all the nucleus; in 28\% there was partial hypoplasia, generally confined to the inferior two thirds and in $12 \%$ the hypoplasia was unilateral, involving the right portion. In addition, agenesis of the arcuate nucleus was diagnosed in $14 \%$ of the cases, and defective neuronal maturation in $10 \%$ of the victims, all SIDS.

Hypoplasia of the pre-Bötzinger nucleus, with a decreased neuronal number and/or dendritic hypodevelopment of the reticular formation, was found in $25 \%$ of unexplained perinatal deaths and in $8 \%$ of the SIDS.

Hypoplasia of one or more nuclei of the raphe system (obscurus, pallidus, median, magnum, caudal linear raphe nuclei) was seen in $57 \%$ of the SIDS and in $67 \%$ of perinatal victims. Hypodevelopment of other nuclei, such as the hypoglossus, dorsal vagal and tractus solitarii nuclei, was also occasionally found.

In the rostral pons and caudal mesencephalon of $35 \%$ of perinatal deaths, particularly intra-partum deaths, hypoplasia was observed, with a few immature neurons or agenesis of the parabrachial/ Kölliker-Fuse complex.

Hypoplasia of the parafacial/facial complex, with a decreased neuronal density and area, was diagnosed in $60 \%$ of unexplained stillbirths. Neuronal cell bodies of the hypoplastic nuclei were small and lengthened, with a flattened nucleus.

Besides, hypodevelopment of other nuclei (hypoglossal, dorsal vagal, tractus solitarii, inferior olivary nuclei) was occasionally observed.

In the cerebellum, histological examination showed an immature structure of the cerebellar cortex, uniformly made up of small, round cells without the usual four-layered shape, in $20 \%$ of late unexplained perinatal deaths and in $26 \%$ of SIDS victims.

In the spinal cord, various degrees of hypodevelopment (neuronal immaturity in a normal structure/hypoplasia/agenesis) of the intermediolateral nucleus were found in $45 \%$ of the victims of unexplained death.

Fig. 1 summarizes the incidence of the main developmental alterations.

- Functional alterations

Using immmunohistochemical methods with specific antibodies for the different neurotransmitters, an altered expression of somatostatin, that is widely present in brainstem nuclei during fetal life but scarcely expressed after birth, was highlighted in the hypoglossus nucleus. Despite a normal structure, this nucleus was in fact somatostatin-immunonegative in $27 \%$ of the unexplained fetal deaths and, on the contrary, immunopositive in $44 \%$ of SIDS victims. A decreased serotonin synthesis in the neuronal cell bodies and fibers of the raphe nuclei was observed in $30 \%$ of the victims.

Negative expression of tyrosine-hydroxylase ( $\mathrm{TH}$ ), an essential enzyme in catecholamine biosynthesis, was detectable despite a well-structured locus coeruleus (the major brainstem producer of noradrenaline), in $57 \%$ of the perinatal and infant sudden deaths.

Table 3

Number of cases with the different alterations.

\begin{tabular}{|c|c|c|c|c|}
\hline Pathological results & $\begin{array}{l}\text { Unexplained perinatal death } \\
\text { victims }(n=95)\end{array}$ & $\begin{array}{l}\text { Control perinatal death } \\
\text { victims }(n=34)\end{array}$ & $\begin{array}{l}\text { SIDS } \\
(n=140)\end{array}$ & $\begin{array}{l}\text { Control infant deaths } \\
(n=44)\end{array}$ \\
\hline \multicolumn{5}{|l|}{$\begin{array}{l}\text { ANS alterations (hypoplasia/agenesis/delayed maturation)-Total number } \\
\text { Brainstem }\end{array}$} \\
\hline Arcuate nucleus & 49 & 8 & 41 & 10 \\
\hline Pre-Bötzinger complex & 24 & - & 11 & - \\
\hline Raphè nuclei & 64 & 1 & 78 & - \\
\hline Parafacial complex & 57 & - & - & - \\
\hline Parabrachial/Kölliker-Fuse complex & 33 & - & 4 & - \\
\hline Other nuclei (hypoglossal, dorsal vagal, tractus solitarii, inferior olivary) & 15 & - & 18 & 1 \\
\hline Cerebellar cortex & 19 & - & 36 & 3 \\
\hline \multicolumn{5}{|l|}{ Spinal cord } \\
\hline Intermediolateral nucleus & 43 & & 63 & - \\
\hline $\begin{array}{l}\text { CCS alterations (Resorptive degeneration, Mahaim fibers, cartilaginous } \\
\text { metaplasia, islands of conduction tissue in the CF) }\end{array}$ & 18 & 4 & 8 & 9 \\
\hline Atherosclerotic alterations & 51 & 1 & 88 & 3 \\
\hline
\end{tabular}

ANS = autonomic nervous system.

$\mathrm{CCS}=$ cardiac conductions system.

Individual victims may display any combination of these pathological alterations. 


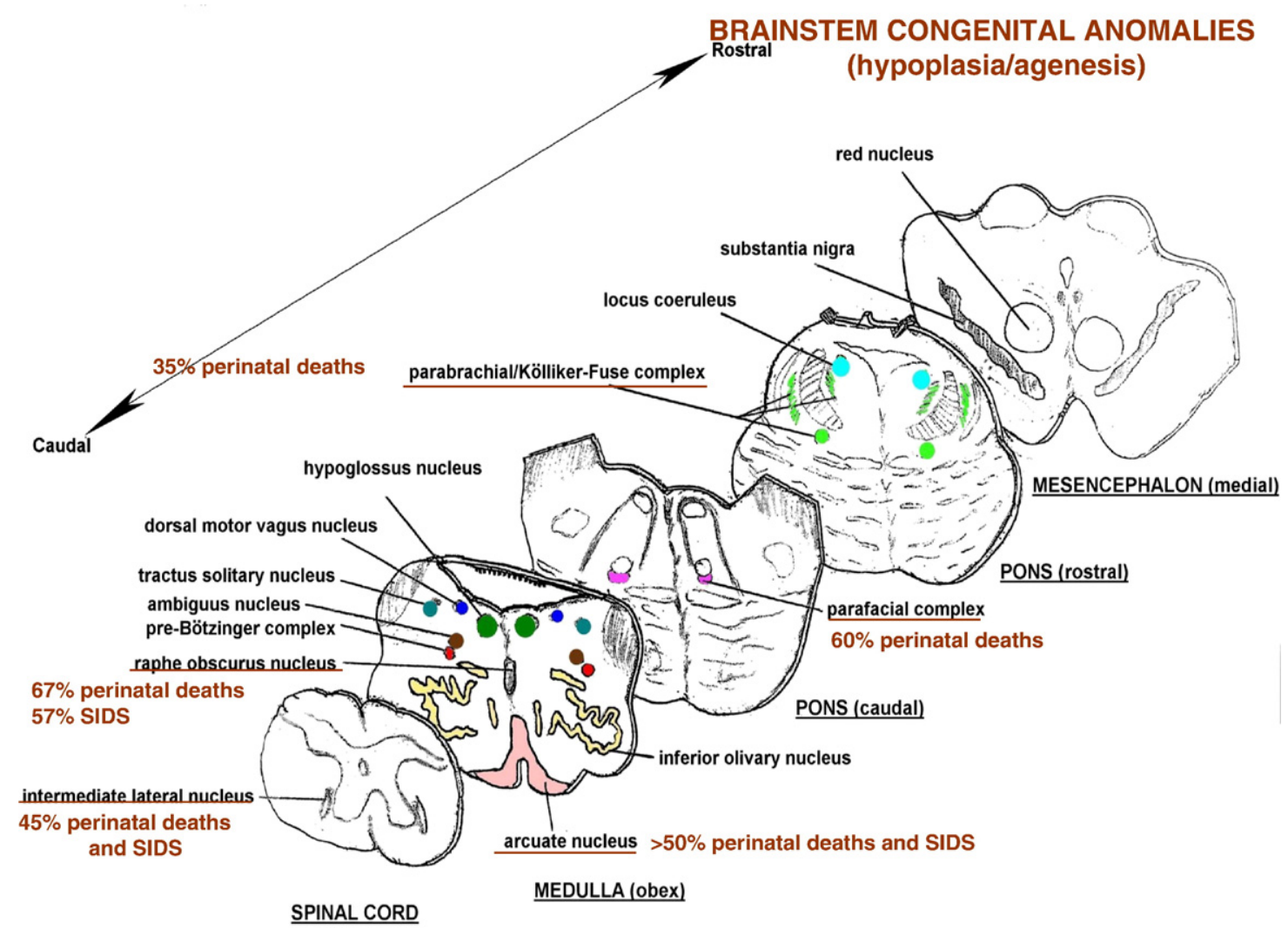

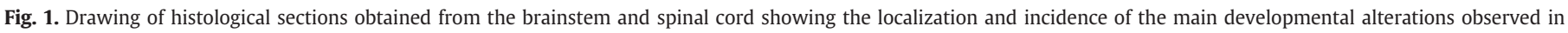
unexplained stillbirths and SIDS.

Unusual apoptosis of the Purkinje cells and of the internal granular layer in the cerebellar cortex was found in $18 \%$ of SIDS victims. A high apoptotic index was observed in the dentate nucleus of $34 \%$ of SIDS cases.

Application of the GFAP method showed the presence of numerous reactive astrocytes in the brainstem and cerebellum of both sudden death victims and controls.

Frequently, above all in fetal deaths, two or more morphological and/or functional alterations were simultaneously present in the same case, such as hypoplasia of the arcuate, pre-Bötzinger and parafacial nuclei and negative somatostatin expression in the hypoglossus nucleus.

- Genetic alterations

Investigation of the polymorphisms of the serotonin transporter (5-HTT) gene, the major determinant of serotoninergic function through the modification of gene transcription and transporter expression, showed that the frequency of the L/L genotype and $\mathrm{L}$ allele was 4 -fold higher in unexplained deaths than in controls. Specifically, the L/L homozygote and S/L heterozygote genotypes were detected in $39 \%$ and $47 \%$ of SIDS cases, respectively. Likewise, the $\mathrm{L} / \mathrm{L}$ and $\mathrm{S} / \mathrm{L}$ genotypes were detected in $30 \%$ and $50 \%$ of stillbirths, respectively. The $\mathrm{L} / \mathrm{L}$ and $\mathrm{S} / \mathrm{L}$ genotype frequencies in controls were $12 \%$ and $47 \%$, respectively. The S/S genotype was detected in $14 \%$ of SIDS, in $20 \%$ of SIUD and in $41 \%$ of control cases, respectively. Therefore, the frequency of the L allele was $62 \%$ in SIDS, $55 \%$ in SIUD and 35\% in controls.

Comparative analysis of the genetic and morphological results indicates a correlation between genotypic serotonin polymorphisms and hypoplasia of the raphe nuclei in the brainstem of unexplained fetal deaths and SIDS victims.

The DNA from all SIDS, SIUD cases and Controls submitted to the homeobox transcription factor PHOX2B testing, showed a PHOX2B 20/20 genotype in all cases, indicating the normal number of 20 alanines on both alleles. These results confirm that none of the victims of sudden death or controls had a mutation compatible with Congenital Central Hypoventilation Syndrome [10].

\subsubsection{Autonomic peripheral nervous system}

Abnormalities were detected in the superior cervical ganglia in 39\% of cases of unexpected perinatal loss and in $22 \%$ of the SIDS. In these, there were SIF (small intensely fluorescent) immature neurons, unipolar or with very poor processes, large juxtaposed bodies and sometimes anastomosed with each other, lacking a satellite-cell mantel. Interneuronal cells, paraganglionic in nature, laden with argentophilic Grimelius positive granules (aminic neurotransmitter) were numerous, occasionally gathered around clusters of immature neurons.

Similar glomus cells were also observed in the capsule of the ganglium nodosum. The mediastinal paraganglia were grossly hypertrophic, with an uneven depletion of the chief-cells neurotransmitter granuli in $24 \%$ of SIDS cases.

\subsection{Neuropathologic findings in controls}

Only hypoplasia of the arcuate nucleus was observed in $23 \%$ of cases, rarely associated to minor hypodevelopment of other brainstem nuclei and delayed maturation of cerebellar and cerebral cortex structures.

\subsection{Cardiovascular pathologic findings in stillbirth versus SIDS}

- Cardiac conduction system

Accessory AV pathways, mainly Mahaim fibers, were detected in $39 \%$ of unexplained perinatal deaths and in $23 \%$ of SIDS victims. Accessory AV communications were uncommon, both of James type, detected in 2 stillbirths, and of Kent type, that is the substrate of WolfParkinson-White ventricular pre-excitation,, detected in 1 stillbirth 
and 2 SIDS victims. Cartilage metaplasia of the fibrous body was detected in $19 \%$ of unexplained perinatal deaths and in $6 \%$ of the SIDS.

- Arterial walls

In $54 \%$ of fetuses and in $63 \%$ of neonatal deaths and SIDS victims, structural lesions of the arterial wall were evident from the 35th week of gestation. The development of atherosclerosis in fetuses is characterized by foci of gross subversion of the tunica media with fragmentation of the smooth muscle cells (SMCs), perpendicularly oriented and infiltrating the intima. After birth there was progressive myointimal thickening, caused by lipids and glycosaminoglycans deposits synthesized by SMCs and by the infiltration of monocytes. These lesions progressed to the formation of common atherosclerotic plaques, already recognizable in infants after few weeks of life.

These atherosclerotic alterations were systemic; they were frequently observed in coronary arteries, mainly in the anterior descending branch, in the carotid, cerebral arteries, aorta and also in mediumsized arteries, including the arteries supplying the cardiac conduction system, and in the arterial walls of the fetal adnexa. Fibromuscular hyperplasia of the pulmonary arteries was also a frequent finding.

\section{Discussion}

The problem of unexpected perinatal loss and the SIDS at post-mortem examination is a Gordian knot that needs to be untied, not cut. Pathologic data on the SIDS, beginning from the so-called thymus-death, a possible lethal thymus-lymphatic state marked by gland enlargement, occasionally related to stenotic respiratory diseases, has been accumulating since 1800 . From the 1970s, inflammatory respiratory diseases, namely pneumonia, bronchiolitis, tracheo-bronchitis, from bacterial or viral causes, and myocarditis, particularly viral, are described among the possible causes and concauses of the SIDS [19].

A relevant growth of knowledge on the anatomical substrates of sudden perinatal loss and the SIDS has been gained from studies of the autonomic nervous system.

Early studies on neuropathology were conducted by Naeye in 1976 [20], who described the presence of brainstem astrogliosis in half of his SIDS cases. This alteration was again emphasized by Kinney et al. [21] in $22 \%$ of the SIDS victims examined.

Subcortical leukomalacia in the white matter [22], developmental delay of dendritic spines and synapses [23], a decreased number of myelinated vagal fibers [24], increased apoptosis in brainstem nuclei [25] and abnormalities in neurotransmitters [26] have all been reported in the SIDS in subsequent research.

All these lesions, albeit non-specific, can be attributed to chronic or repeated hypoxia complicating sleep apnea and alveolar hypoventilation, suggesting the possibility that abnormal development of the neuronal circuitry between the brainstem centers that regulate rhythmic breathing and arousal gives rise to cardiorespiratory instability. This concept provides an insight into the specific mechanisms leading to unexpected death.

A variety of abnormalities is also found in the brains of stillborns, the most common being brainstem and spinal cord necrosis, periventricular leukomalacia, gliosis, intraventricular hemorrhage, cerebral infarcts [2].

No cause of death was instead identified after autopsy in unexpected fetal death [27].

\section{Neuropathology of stillbirth versus SIDS}

While the data reported in the literature in unexpected perinatal loss and SIDS refer to non-specific alterations of the autonomic nervous system, our investigations in this field have contributed to reveal the localization and the nature of a variety of specific neuronal congenital anomalies, particularly of the brainstem, spinal cord and cerebellum.

From the overall analysis of the neuropathologic results, the following prominent data emerged:
- in $92 \%$ of sudden perinatal and infant deaths one or more congenital morphological and/or functional abnormalities of the autonomic nervous system are present. These alterations are more numerous in unexplained antepartum deaths as compared with intra-partum, neonatal deaths and the SIDS;

- ante-partum deaths are characterized by the association of many developmental alterations of the brainstem, cerebellum and spinal cord structures; an exclusive alteration in these deaths is hypoplasia of the facial/parafacial complex;

- intra-partum deaths are characterized by a decreased number of nuclei shown to be involved in developmental disturbances, and by the high frequency of hypoplasia of the parabrachial/KöllikerFuse complex;

- in SIDS victims the main alteration is hypoplasia of the arcuate nucleus, present in over $50 \%$ of victims, sometimes associated to hypoplasia of other nuclei such as the hypoglossus, the preBötzinger, the tractus solitarii, the inferior olivary nuclei;

- a frequent finding in both unexpected perinatal death and the SIDS is defective synthesis of neurotransmitters (catecholamines in the locus coeruleus, serotonin in the raphe nuclei, somatostatin in the hypoglossus nucleus);

- there is a high incidence of genetic polymorphisms, such as the serotonin L/L genotype, present in 39\% of SIDS cases and in 30\% of sudden fetal deaths, frequently related to morphological hypodevelopment of one or more nuclei of the raphe complex.

A common finding in both unexplained perinatal death and the SIDS was hypoplasia of the arcuate nucleus, a component of the ventral surface of the medulla oblongata which participates in sleeprelated homeostatic responses including chemoreception, thermoregulation, respiratory drive and breathing, arousal, reflex regulation of blood pressure and cardiovascular responses [12,13,28].

Arcuate nucleus hypoplasia is frequently associated to hypoplasia of the pre-Bötzinger nucleus, defined as a group of neurons of the ventrolateral medulla, that are essential for generating the respiratory rhythm, as well as for modulating eupneic breathing [14,29]. Instead, the Kölliker-Fuse nucleus was found to be hypodeveloped particularly in sudden intra-partum deaths; it has an important function during intrauterine life, inhibiting the response of central and peripheral chemoreceptors (which are already fully formed and potentially functional in the last weeks of pregnancy) and therefore any respiratory reflex. After birth, the Kölliker-Fuse abruptly reduces its inhibitory effects and becomes active as a respiratory center able to coordinate the pulmonary motor responses to hematic oscillations of $\mathrm{pO}_{2}, \mathrm{pCO}_{2}$ and $\mathrm{pH}$ [15].

In addition, exclusively in unexplained stillbirths, hypoplasia of the parafacial nucleus, consisting of "pre-inspiratory" neurons that periodically trigger the inspiratory neurons of the pre-Bötzinger complex, was very frequent. Therefore, the function of hierarchical modulation of the breathing circuitry can be ascribed to the parafacial nucleus [16].

Among the functional alterations, defective expression of neurotransmitters was diagnosed in the brainstem, particularly of catecholamines in the locus coeruleus, serotonin in the raphe complex and somatostatin in the hypoglossus nucleus, besides altered apoptotic programs in the cerebellum, and precisely in the Purkinje and internal granular layers of the cerebellar cortex as well as in the dentate nucleus.

A notable finding in this study was the increased frequency of the serotonin L allele not only in SIDS cases, in agreement with previously published data [9], but also in unexplained stillbirths, never considered in relation to serotonin network dysfunctions [30]. An additional observation was the significant correlation between the $\mathrm{L}$ allele and hypoplasia of the raphe nuclei. In fact, a high percentage of sudden fetal and infant deaths with the $\mathrm{L} / \mathrm{L}$ or $\mathrm{L} / \mathrm{S}$ genotype showed hypoplasia of one or more nuclei of both the superior and inferior raphe groups, 
suggesting involvement of a serotonin polymorphism in hypodevelopment of the raphe system. It is known, in fact, that the serotonin system plays a trophic role during neuronal development in the fetal brain. A dysfunction in serotonergic transmission during intrauterine life could lead to hypodevelopment of the neuronal structures checking vital functions, with a fatal outcome.

\section{Cardiovascular pathology of stillbirth versus SIDS}

Unexpected perinatal loss and the SIDS are also significantly associated with developmental disturbances of the cardiovascular system.

Sudden death can occur due to changes in the cardiac action, mostly manifesting with arrhythmias, that may be caused by microscopic malformations of the conduction system. The finding of accessory AV communications, particularly nodo-fascicular ventricular bundles (Mahaim fibers) is quite frequent in perinatal unexplained loss, as also in SIDS (but a clinicopathologic assessment of their lethal arrhythmogenic potential is often impossible). These congenital abnormalities, under particular conditions and/or neurovegetative stimuli, are liable to provoke electrical dyshomogeneity, instability and desynchronization, raising the risk of malignant functional arrhythmias.

In our study, accessory AV pathways, mainly Mahaim fibers, were detected in $39 \%$ of unexpected perinatal deaths and in $23 \%$ of SIDS victims. Accessory AV communications of James and Kent type the substrate of Wolf-Parkinson-White pre-excitation, were uncommon, being detected in only 2 unexpected perinatal deaths and in 2 SIDS victims, respectively.

These lesions have been attributed to the variable outcome of a "resorptive degeneration" process that normally "reshapes" the functional pathways in the fetal and early neonatal period; theoretically, if this process is defective, anomalous AV connection may persist, whereas, if it is exaggerated, it could result in blocking disruption. Clinically, this abnormality can present without hemodynamic impairment, while manifesting with high-risk reciprocating arrhythmias.

In addition, the central cardiac structure "supporting" the cardiac system could possibly interface with the conduction of impulses, as in the cases of cartilaginous metaplasia of the fibrous body.

The most frequent observations in the vascular system of sudden perinatal deaths and SIDS are arterial atherosclerotic lesions observed in $54 \%$ of fetuses from the 35th gestational week and in $63 \%$ of neonatal deaths and SIDS victims. The atherosclerosis is systemic in nature and in the prenatal period, involvement of the fetal adnexa arteries is common. By thickening the arterial wall, the atherosclerotic lesions can reduce the placental flow and reduce fetal oxygenation. Finally, serious alterations of the pulmonary arteries were observed in $7 \%$ of the sudden perinatal deaths and the SIDS.

\section{Exogenous-environmental risk factors}

Among the causes which trigger and/or promote unexplained death, exogenous risk factors that alter the intrauterine environment are relevant,such as infections (pulmonary virosis with respiratory impairment), familial tobacco smoking, maternal drug abuse, maternal alcoholism and likely atmospheric pollution.

In particular, exposure to tobacco smoke in utero is the most important preventable risk factor. Smoking, by affecting fetal oxygenation, causes vasoconstriction and reduces the fetal blood flood, as well as inducing placental atherosclerosis

In addition, air pollution, particularly in the Lombardy Region, where our studies have been prevalently conducted, which features high rates of both gases (carbon monoxide, nitrogen dioxide, ozone, sulphur dioxide) and particulate matter (above all $\mathrm{PM}_{10}$, with a median diameter of $<10 \mu \mathrm{m}$ ), could have an important influence in determining sudden unexplained perinatal and infant death. We postulate that gas pollutants in particular, like cigarette smoke, can cross the placenta during pregnancy through the maternal blood, and lead to a hypoxic status responsible for structural and/or functional impairments of the central nervous system.

Finally we point out that maternal and antenatal risk factors could yield phenotypes susceptible to sudden and unexpected death in perinatal and infant life in the presence of protein products produced by a specific polymorphism and/or the mutation of genes affecting brainstem autonomic control, i.e., the same risk factors could have very different effects in subjects with a different genetic constitution. All the morphological and/or functional alterations reported up to now can be interpreted as the consequence of these interactions.

\section{Nosology}

The observation of similar anomalies of the autonomic nervous system and of the cardiac conduction system in both unexplained perinatal deaths and SIDS indicates their common congenital nature and supports the statement in the National Institute of Child Health and Development SIDS Strategic Plan 2000 that "SIDS is a developmental disorder. Its origins are during fetal development" [31]. These observations supported a new holistic approach to the SIDS, analogically linked with unexpected perinatal loss.

The developmental anomalies and genetic substrates common to unexpected stillbirth, early neonatal death and SIDS suggest that the definitions of these deaths, currently nosographically distinct according to the International classification of disease (ICD) [32], should be unified.

The need to perform a complete autopsy of all victims of these unexpected deaths is unanimously recognized, since prevention of these diseases will be based mostly on a better recognition of the abnormalities found in various organs, as well as on the individuation of the basic underlying mechanisms. Since this problem is highly complex, it is extremely important to obtain an exhaustive diagnosis of each case, together with full details of the familial and circumstantial background to the demise. The scientific advantages derived from a better understanding of perinatal loss and SIDS are difficult to evaluate, but are certainly extremely significant. Such an understanding would encompass the prenatal period, which is now regarded as the background to many responses to disease in infants, adults and even elderly patients.

\section{Conflict of interest statement}

All Authors declare that they have no conflicts of interest, financial or otherwise to declare.

\section{Funding}

No financial assistance was received to support the study.

\section{Acknowledgments}

The authors thank Dr. Graziella Alfonsi for her precious technical assistance and Dr. Mary Victoria Candace Pragnell, B.A. for English language assistance.

\section{References}

[1] World Health Organization (WHO). Neonatal and perinatal mortality: country, regional and global estimates; 2006. p. 1-75.

[2] Grafe MR, Kinney HC. Neuropathology associated with stillbirth. Semin Perinatol 2002;26:83-8.

[3] Bell JE, Becker JC, Wyatt B, Keeling JW, McIntosh N. Brain damage and axonal injury in a Scottish cohort of neonatal deaths. Brain 2005;128:1070-81.

[4] Squier M, Keeking JW. The incidence of prenatal brain injury. Neuropathol Appl Neurobiol 1991;17:29-38. 
[5] Sparks DL, Hunsaker JC. Neuropathology of sudden infant death (syndrome). Childs Nerv Syst 2002;18:568-92.

[6] Krous HF. Instruction and reference manual for the International Standardise Autopsy Protocol for sudden unexpected infant death. J SIDS Infant Mortal 1996;1: 203-46.

[7] Matturri L, Ottaviani G, Lavezzi AM. Techniques and criteria in pathologic and forensic-medical diagnostics of sudden unexpected infant and perinatal death. Am J Clin Pathol 2005;124:259-68.

[8] Matturri L, Ottaviani G, Lavezzi AM. Guidelines for neuropathologic diagnostics of perinatal unexpected loss and sudden infant death sindrome (SIDS). A technical protocol. Virchows Arch 2008;452:19-25.

[9] Weese-Mayer DE, Zhou L, Berry-Kravis EM, Maher BS, Silvestri JM, Marazita ML. Association of the serotonin transporter gene with the Sudden Infant Death Syndrome: a haplotype analysis. Am J Med Genet 2003;122A:238-45.

[10] Weese-Mayer DE, Berry-Kravis EM, Ceccherini I, Rand CM. Congenital centra hypoventilation syndrome (CCHS) and sudden infant death syndrome (SIDS): kindred disorders of autonomic regulation. Review. Respir Physiol Neurobio 2008; 164:38-48.

[11] Matturri L, Giuliani P, Scarpato M, Bonani L, Mecchia D, Lavezzi AM. Italian National Data Bank of Stillbirth versus SIDS. Public Health Rep 2010;125:516.

[12] Matturri, Biondo B, Mercurio P, Rossi L. Severe hypoplasia of medullary arcuate nucleus: quantitative analysis in sudden infant death syndrome. Acta Neuropathol (Berl) 2000;99:371-5.

[13] Matturri, Minoli I, Lavezzi AM, Cappellini A, Ramos S, Rossi L. Hypoplasia of medullary arcuate nucleus in unexpected late fetal death (stillborn infants): a pathologic study. Pediatrics 2002;109:E43.

[14] Lavezzi AM, Matturri L. Functional neuroanatomy of the human pre-Bötzinger complex with particular reference to sudden unexplained perinatal and infant death. Neuropathology 2008;28:10-6.

[15] Lavezzi AM, Ottaviani G, Ballabio GM, Rossi L, Matturri L. Preliminary study on the cytoarchitecture of the human parabrachial/Kölliker-Fuse complex with reference to sudden infant death syndrome and sudden intrauterine unexplained death. Pediatr Dev Pathol 2004;7:171-9.

[16] Lavezzi AM, Matturri L. Hypoplasia of the parafacial/facial complex: a very frequent finding in sudden unexplained fetal death. Open Neurosci J 2008;2:1-5.

[17] Lavezzi AM, Ottaviani G, Mauri M, Matturri L. Alterations of biological features of the cerebellum in sudden perinatal and infant death. Curr Mol Med 2006;6:429-35.
[18] Matturri, Ottaviani G, Lavezzi AM, Rossi L. Early atherosclerotic lesions of the cardiac conduction system arteries in infants. Cardiovasc Pathol 2004;13:276-81.

[19] Bergman AB. Synthesis. In: Bergman AB, Beckwith JB, Ray CG, editors. Sudden Infant Death Syndrome. Seattle, WA: University of Washington Press; 1970. p. 210-1.

[20] Naeye RL. Brain-stem and adrenal abnormalities in the sudden-infant-death syndrome. Am J Clin Pathol 1976;66:526-30.

[21] Kinney HC, Burger PC, Harrell FE, Hudson RP. "Reactive gliosis" in the medulla oblongata of victims of the SIDS. Pediatrics 1983;72:181-7.

[22] Takashima S, Armstrong D, Becker LE, Huber J. Cerebral white matter lesions in the sudden infant death syndrome. Pediatrics 1978;62:155-9.

[23] Quattrochi PT, McBride P, Yates AJ. Brainstem immaturity in sudden infant death syndrome: a quantitative rapid Golgi study of dendritic spines in 95 infants. Brain Res 1985;325:39-48.

[24] Kinney HC, Brody BA, Finkelstein DM, Vawter GF, Mandell F, Gilles FH. Delayed central nervous system myelination in the sudden infant death syndrome. J Neuropathol Exp Neurol 1991;50:29-48.

[25] Waters KA, Meehan B, Huang JQ, Michaud J, Cote A. Neuronal apoptosis in sudden infant death syndrome. Pediatr Res 1999;45:166-72.

[26] Ozawa Y, Takashima S. Developmental neurotransmitter pathology in the brainstem of sudden infant death syndrome: a review and sleep position. Forensic Sci Int 2002;130:53-9.

[27] Magee JF. Investigation of stillbirth. Pediatr Dev Pathol 2001:4:1-22.

[28] Filiano JJ, Kinney HC. Arcuate nucleus hypoplasia in the sudden infant death syndrome. J Neuropathol Exp Neurol 1992;51:394-403.

[29] Rekling JC, Feldman JL. PreBötzinger complex and pacemaker neurons: hypothesized site and kernel for respiratory rhythm generation. Annu Rev Physiol 1998;60:385-405

[30] Lavezzi AM, Casale V, Oneda R, Weese-Mayer DE, Matturri L. Sudden infant death syndrome and sudden intrauterine unexplained death: correlation between hypoplasia of raphé nuclei and serotonin transporter gene promoter polymorphism. Pediatr Res 2009;66:22-7.

[31] NICHD Staff. The sudden infant death syndrome strategic plan 2000. Available at: www.nichd.nih.gov Accessed March 20, 2002.

[32] World Health Organization. International Statistical Classification of Disease and related health problems. Geneva: WHO; 1993. 1oth revision (ICD-10). 